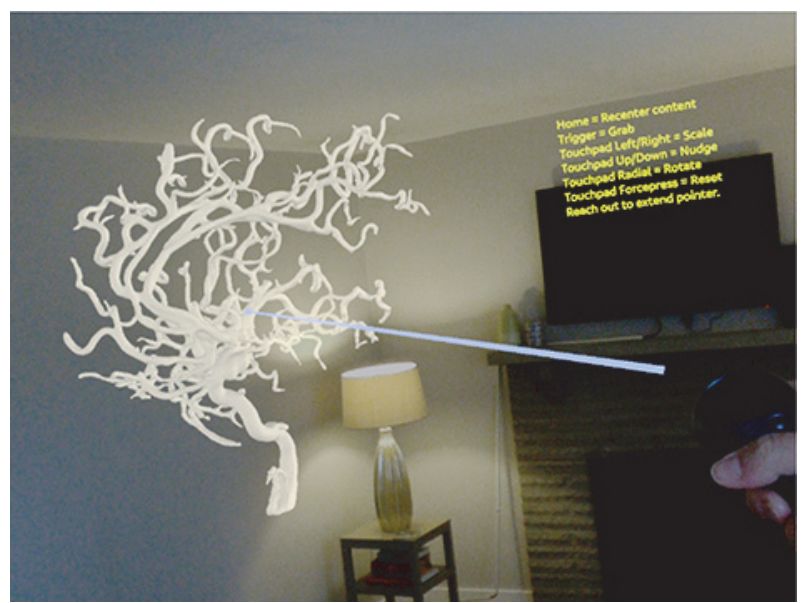

Abstract E-195 Figure 1

such as traditional angiographic monitors, holograms, virtual or augmented reality models or $3 \mathrm{D}$ printed models. Users are able to experience realistic $3 \mathrm{D}$ virtual elements with augmented depth perception and binocular field of view. Newer technology has now allowed for interaction with 3D objects within these virtual environments through the use of hand gestures or hand controllers. The authors describe our preliminary experience with $\mathrm{MxR}$ as an adjunct tool to traditional angiographic imaging in the preprocedural workup of patients with complex aneurysms using a novel spatial computer, the Magic Leap One device (Magic Leap, Inc Plantation, Florida) which uses a virtual retinal display, which superimposes 3D computer-generated imagery over real world objects, by projecting a digital light field into the user's eye.

Materials and Methods Tomographic Angiographic (DynaCT) data was imported and segmented to create $3 \mathrm{D}$ meshes of the intracranial vasculature. The $3 \mathrm{D}$ meshes were then projected into MxR space, allowing the operator to inspect the vasculature using a MxR headset (Magic Leap) as well as interact with the aneurysm and adjacent vessels (handling, rotation, magnification, and sectioning) using hand gestures or hand controllers.

Results 3D segmentation of a complex aneurysms was successfully performed and projected into MxR. Conventional and MxR visualization modes were equally effective in identifying and classifying the pathology. MxR visualization allowed the operators to manipulate the dataset to achieve a greater understanding of the anatomy of the parent vessel, the angioarchitecture of the aneurysm, and the surface contours of all visualized structures.

Conclusion This preliminary study demonstrates the feasibility of utilizing MxR for preprocedural evaluation in patients with anatomically complex neurovascular disorders. This novel visualization approach may serve as a valuable adjunct tool in deciding patient-specific management, including decisions on prognostication and open surgical and endovascular treatment options.

Disclosures G. Deib: None. D. Smith: None. B. Chaudhary: None. S. Boo: None. A. Tarabishy: None. J. Carpenter: None. A. Rai: None.

\section{E-196 THE RISK OF ISCHEMIA IN PREVENTIVE TEMPORARY ARTERY OCCLUSION OF THE CONTRALATERAL ANTERIOR CEREBRAL ARTERY DURING MICROSURGICAL CLIPPING OF ACOM ANEURYSMS}

${ }^{1} \mathrm{C}$ Wipplinger*, ${ }^{2} \mathrm{~F}$ Mrosk, ${ }^{1} S$ Tülü, ${ }^{1} \mathrm{C}$ Preuss-Hernandez, ${ }^{1} \mathrm{~W}$ Ho, ${ }^{1} \mathrm{~A}$ Görke, ${ }^{1} \mathrm{M}$ Ortler, ${ }^{1} \mathrm{O}$ Petr, ${ }^{1} \mathrm{C}$ Thomé. ${ }^{1}$ Department of Neurosurgery, Medical University of Innsbruck, Innsbruck, Austria; ${ }^{2}$ Department of Neurosurgery, Charité-Universitätsmedizin Berlin, Berlin, Germany

\subsection{6/neurintsurg-2020-SNIS.227}

Introduction Temporary artery occlusion (TAO) is commonly used to facilitate dissection and clipping of intracranial aneurysms. When clipping anterior communicating artery (Acom) aneurysms, a possible strategy is to perform early preventive TAO of the contralateral A1 segment of the anterior cerebral artery before dissecting towards the aneurysm. In case of intraoperative complications such as complicated aneurysm dome preparation or inadvertent rupture of the aneurysm, full proximal control can be quickly gained by additional TAO of the easily accessible ipsilateral A1. Also, right-sided approaches are sometimes preferred due to reduced risk of damage of the dominant hemisphere and right-handedness of the surgeon. However, this may lead to prolonged TAO times of the contralateral A1 and might involve the risk of cerebral ischemia, especially if the contralateral A1 is the dominant supplier. In the present study, we aimed to investigate the safety of this strategy.

Methods We retrospectively analyzed the clinical and imaging outcomes of Acom aneurysms treated at our institution over a period of seven years by a right-sided frontotemporal approach. Temporary contralateral A1 occlusion early after opening of the chiasmatic cistern was defined as early TAO. We evaluated the total TAO time, A1 dominance, intraoperative rupture rates as well as postoperative mRS. The primary outcome parameter was postoperative ischemia determined on CT scans within 48 hours after surgery by a board-certified neuroradiologist.

Results A total of 81 including 52 ruptured and 29 unruptured aneurysms were treated by microsurgical clipping over a period of seven years. In 48 patients (59\%) early contralateral A1 TAO was performed for an average of $22 \pm 20 \mathrm{~min}$ and the majority of these cases (30 patients) harbored a dominant contralateral A1. Bilateral TAO was performed in 22 patients $(27 \%)$ for $11 \pm 10 \mathrm{~min}$. Intraoperative rupture occurred in 20 patients $(25 \%)$ of which 10 received early TAO. Postoperatively, 5 patients showed postoperative ischemia (6\%). Bilateral TAO as well as dominant early TAO showed no significant correlation with ischemia or worse post-operative mRS. However, patients with ischemia had a significantly longer overall TAO time $(45 \pm 27$ mins) than patients without (19 \pm 18 mins) $(\mathrm{p}=0.02)$.

Conclusion In our patient population neither early TAO of the dominant A1 nor bilateral TAO appeared to have an increased risk for ischemia. However, prolonged TAO, regardless of which side should be avoided.

Disclosures C. Wipplinger: None. F. Mrosk: None. S. Tülü: None. C. Preuss-Hernandez: None. W. Ho: None. A. Görke: None. M. Ortler: None. O. Petr: None. C. Thomé: None. 